\title{
Cómo la Macaronesia ha influido en nuestra perspectiva sobre los ecosistemas insulares
}

\author{
A. M. C. Santos ${ }^{1,2,3, *}$, M. Florencio ${ }^{3,4}$, S. Nogué ${ }^{5,6}$, J. Patiño $0^{3,7}$, A. Traveset ${ }^{8}$, P. A.V. Borges ${ }^{3}$ \\ (1) Departamento de Biogeografía y Cambio Global, Museo Nacional de Ciencias Naturales (CSIC), 28006 Madrid, España. \\ (2) Grupo de Ecologia Forestal y Restauración, Departamento de Ciencias de la Vida, Universidad de Alcalá, 28805 Alcalá de Henares, Madrid, España. \\ (3) cE3c - Centre for Ecology, Evolution and Environmental Changes / Azorean Biodiversity Group y Universidade dos Açores - Departamento de Ciências \\ e Enhenharia do Ambiente, 9700-042 Angra do Heroísmo, Terceira, Azores, Portugal. \\ (4) Departamento de Ecologia, Instituto de Ciências Biológicas, Universidade Federal de Goiás, Goiânia, Brazil. \\ (5) Geography and Environment, University of Southampton, SO17 1BJ, Southampton, Reino Unido. \\ (6) Oxford Long-Term Ecology Laboratory, Department of Zoology, University of Oxford, OX1 3PS, Oxford, Reino Unido. \\ (7) Island Ecology and Evolution Research Group, Instituto de Productos Naturales y Agrobiología (IPNA-CSIC), Tenerife, España. \\ (8) Instituto Mediterráneo de Estudios Avanzados, IMEDEA (CSIC-UIB), 07190-Esporles, Mallorca, España.
}

* Autor de correspondencia: A.M.C. Santos [ana-margarida.c.santos@gmail.com]

> Recibido el 12 de agosto de 2016 - Aceptado el 23 de septiembre de 2016

Santos, A.M.C., Florencio, M., Nogué, S., Patiño, J., Traveset, A., Borges, P.A.V. 2016. Cómo la Macaronesia ha influido en nuestra perspectiva sobre los ecosistemas insulares. Ecosistemas 25(3): 166. Doi.: 10.7818/ECOS.2016.25-3.25

Actualmente el estudio de los ecosistemas insulares está pasando por un momento de constantes avances, siendo muchas de las nuevas y relevantes aportaciones fruto de estudios desarrollados en la Macaronesia (Azores, Canarias, Cabo Verde y Madeira). Ésta ha sido la mayor motivación para que el IBIG (Island Biology Interest Group - grupo de interés especial dentro de la AEET y de la SPECO) organizara un simposio dedicado a la Macaronesia, dentro del congreso internacional "Island Biology 2016 - II International Conference on Island Evolution, Ecology and Conservation", realizado entre los días 18 y 22 de Julio, en Angra do Heroismo (isla Terceira, Azores), Portugal. El objetivo principal del simposio ha sido presentar una visión general de la investigación pasada y presente desarrollada en la región Macaronésica, colocándola en el contexto general de la biogeografía de islas.

En el simposio participaron diez ponentes, cuyas comunicaciones representaron bien los distintos archipélagos, grupos taxonómicos, y áreas del conocimiento de la Macaronesia. Después de que Ana M. C. Santos presentara el IBIG así como los objetivos del simposio, tuvo lugar la ponencia principal por parte de José María Fernández-Palacios, quien explicó el contexto histórico, geográfico, geológico y biológico de la región Macaronésica, así como el por qué de su importancia dentro de la biogeografía de islas, ofreciendo también un visión global de las expediciones científicas clásicas. Sofia Gabriel mostró los eventos de colonización del ratón doméstico (Mus musculus domesticus) en la isla de Madeira, desvelados a raíz del estudio de distintas razas cromosómicas. Rui Nunes realizó una ponencia sobre el efecto de borde en el recambio espacial y temporal de artrópodos de los bosques nativos de Azores. Tiago Rodrigues abordó la dispersión en aves (Gallinago spp.), mostrándonos la existencia de flujo genético entre las poblaciones de Azores y las del Paleártico. Jairo Patiño presentó patrones de colonización y especiación de la flora de briófitos de Macaronesia, revisando los mecanismos evolutivos principales, así como sus diferencias con respecto a angiospermas. Alfredo Valido evidenció las consecuencias genéticas y demográficas de la pérdida de origen antrópico de lagartos frugívoros en Canarias sobre una planta arbustiva (Neochamaelea pulverulenta), cuyas semillas son dispersadas por este grupo endémico de lagartos. Jaume Seguí nos presentó un estudio sobre los impactos de la herbivoría y las variables abióticas asociadas a un gradiente altitudinal sobre la violeta del Teide (Viola cheiranthifolia), una planta perenne endémica de Tenerife. Juan Carlos Illera hizo una revisión de los estudios sobre la relación parásito-hospedador en la Macaronesia, enfocándose principalmente en lagartos y aves. Raquel Vasconcelos nos mostró el estado actual del conocimiento sobre la diversidad taxonómica y genética de los reptiles de Cabo Verde, abordando su conservación. Finalmente, Martin Wiemers describió la diversidad de mariposas de Canarias y Madeira.

Este evento demostró la diversidad de aproximaciones al estudio de la ecología, evolución y conservación en islas, poniendo en evidencia la importancia y el potencial futuro de seguir investigando en la región Macaronésica. Para acceder a los resúmenes del simposio se puede consultar la revista Arquipélago (suplemento 9, 2016, http://www.islandbiology2016.uac.pt/). 